\title{
The cutaneous saltatory area and its presumed neural basis
}

\author{
FRANK A. GELDARD and CARL E. SHERRICK \\ Princeton University, Princeton, New Jersey
}

\begin{abstract}
The saltatory area, defined as that region of the skin within which two successive and spatially separated signals will interact to produce apparent mislocalization of the prior signal, is measured by a campimetric technique applied to the palm, finger pad, and volar forearm. As contrasted with earlier measures on larger anatomical units, saltatory areas of the hand region are considerably compressed. Comparisons between cortical receptive fields and saltatory areas lead to speculation concerning the operation of the neural principle of "magnification" in the establishment of saltatory limits. The correspondence of configurational properties of both receptive fields and saltatory areas suggests a commonality of principles underlying both, but demands more information on the temporal dimensions of cortical interaction to confirm the parallelism.
\end{abstract}

The cutaneous saltatory effect is one in which a pulse delivered to the skin by mechanical, electrical, or even thermal means will be mislocalized if a second stimulus appears not too far away in either time or space. It has been under investigation for over a decade (Geldard, 1975, 1982; Geldard \& Sherrick, 1972). The phenomenon has been found to be highly reproducible, to follow rigorous rules with respect to its dependence on certain specific variables, and to have close analogs in the companion channels of vision and audition.

With respect to the temporal separation of the two stimuli, it appears that any interval between the approximate limits of 20 and $250 \mathrm{msec}$ suffices to produce a phenomenal displacement of the prior stimulus. The excitation created by the first "tap" is apparently held in the nervous system in a metastable condition for a period of about a quarter of a second. If no ensuing stimulus appears, the initial arousal process evolves a focus and gets firmly established, so to speak. If, on the other hand, subsequent stimulation at a nearby locus occurs within the prescribed time interval, the first stimulus will appear to be displaced towards the second one: a relatively great distance if the time is short, only slightly if the time is long. If the interval is as little as $20 \mathrm{msec}$ or thereabouts, the first tap will seem to coalesce with the second at the perceived locus of the second, a condition we have called "coincidence." The minimal perceived departure of the initial stimulus from its

This research was supported by National Institutes of Health Grant NS 04755 to Princeton University. Requests for reprints should be sent to the authors at: Cutaneous Communication Laboratory, C-336 E-Quad, Princeton University, Princeton, New Jersey 08544 . "true" locus, which appears at intervals of about $250 \mathrm{msec}$, has been dubbed "exodus."

So much for the necessary temporal separation of the two signals, which appears to be unaffected by rather large variations in the qualitative, intensive, and extensive aspects of the stimuli. What of their spatial relations? A master generalization is not possible here, for the limits of saltatory displacement are far from uniform in different regions of the body and different orientations of the two stimuli. Indeed, it has been found that substitution of a short train of pulses for the initial tap greatly expands the zone in which saltation will operate (Geldard, 1982, pp. 164 165). In view of this, if different body regions are to be tested for their saltatory potential, it is important that a uniform technique of exploration be applied to all skin samples. The results of employing such a procedure are now at hand. The limits of saltatory displacement in four areas-anterior and posterior thigh, dorsal forearm, and ventral thorax-have been previously reported (Geldard, 1982, pp. 155162). All these were the sites of campimetric measurement in which a three-tap sequence of brief (5$\mathrm{msec}$ ) haversine pulses was employed: $P_{1}$, delivered to the first locus $\left(\mathrm{L}_{1}\right)$ to supply a localizing cue; a temporal silent interval of $800 \mathrm{msec}$ (optimal for mobilizing attention but well outside the saltatory range of times); $P_{2}$, providing the tap that is subject to displacement, also delivered at $L_{1}$ and identical with $P_{1}$ in every respect; $P_{3}$, the "attractant," matched to $\mathrm{P}_{2}$ for loudness and presented at a variable second locus $\left(\mathrm{L}_{2}\right)$ after a manipulable delay of 20 $300 \mathrm{msec}$.

The present paper extends the determination of saltatory limits to the volar forearm, palm, and pad of the index finger. There are some surprises in the outcomes. The regions previously measured had been 
selected partly for the relative ease with which apparatus could be accommodated by them, partly for the absence of underlying bone (excellent conductors of forced vibrations and thus generators of misleading localizations), and partly for freedom from interference by respiratory and circulatory events. All are, in general, hairy and sparsely innervated. Two of the areas involved here, palm and finger, are, by contrast, glabrous and much more densely innervated. The third, the volar forearm, stands somewhat midway between palm and dorsal forearm in several respects.

Experiments were completed in the sequence of palm, finger pad, and volar forearm, and they will be reported in this order.

\section{Palm}

The human palm presents special problems for experiments involving mechanical stimulation. The sheer circumstances of high mobility of the hand, its dense innervation, and its ubiquitous bony and musculotendinous substrate demand that steps be taken to insure constancy of posture during exploration, to keep stimulus pulse amplitudes very low, and to employ light but steady static force with small contactors. Adequate control in all these respects was achieved. The observer's left arm was supported by an armrest lined with sponge rubber. The hypothenar edge of the hand rested on a padded platform with thumb up, the latter held in a comfortable sling, and the first joints of the fingers engaged a rubbercovered vertical post. The knuckles rested against an adjustable padded support. Contactors were 6mm-long, No. 1-72 (1.8-mm-diam) nylon screws, rounded at the ends and cemented to the distal edges of $9.5-\mathrm{mm}$-wide piezoceramic reed benders (Bimorphs). These imparted a sweeping motion to the skin surface contacted. The Bimorphs were clamped to the lower ends of aluminum pendulums, $12.7 \mathrm{~cm}$ long; their fulcrums were miniature ball bearings. Adjustable brass counterweights controlled pendulum swing and regulated static pressure. The two pendulums were independently adjustable for position in all three dimensions. Contactor static force, after some preliminaries, was set at $3 \mathrm{~g}$ (see Figure 1).

Each Bimorph was supplied with a strain gauge (BLH, Type SR-4 semiconductor) which was incorporated in a dynamic gauge circuit (Sherrick, 1975). The output of the gauges was fed to an oscilloscope; waveform and pulse amplitude, as well as temporal relations between pulses, could thus be monitored during stimulation. Pulse timing was additionally indicated, in milliseconds, on a HewlettPackard 5300A measuring system. The Bimorphs were driven by shaped pulses supplied by waveform and pulse generators of the Tektronix 160 series operating through Langevin power amplifiers. Pulse

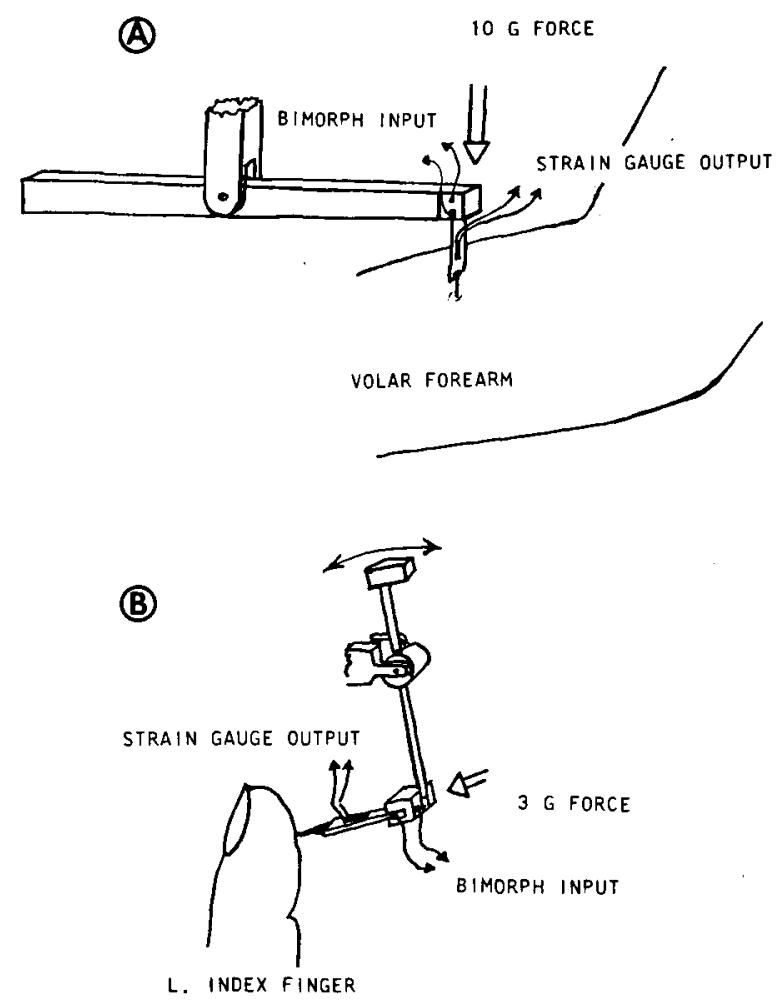

Figure 1. Contactor mountings for the forearm, $A$, and for the fingertip, B. That for the palm is identical with $A$. In all three instances, there is, naturally, a second Identical contactor at variable locus $\left(\mathbf{L}_{2}\right)$.

durations were uniformly set at $5 \mathrm{msec}$, pulse intensities always at $14 \mathrm{~dB}(\mathrm{SL})$, and waveforms of the two channels were matching, smooth haversines.

The timing sequence has been described above $\left(\mathbf{P}_{\mathbf{t}}\right.$ at $L_{1}, 800$-msec silent interval, $P_{2}$ at $L_{1}$, variable $P_{2^{-}}$ $P_{3}$ interval, $P_{3}$ at $L_{2}$ ). A 6.3-sec intertrial period followed. The $P_{2}-P_{3}$ interval, variable from 20 to $300 \mathrm{msec}$ and regulated by a 14-point multiple switch that gave ca 20-msec steps, was under the observer's control. He could sweep the full range of times as frequently as he wished until he was satisfied as to whether $\mathrm{P}_{2}$ remained in its veridical position (coincident with the preceding $P_{1}$ ) or was displaced towards $\mathbf{P}_{\mathbf{3}}$. If saltation was in effect, short $\mathbf{P}_{\mathbf{2}}-\mathbf{P}_{\mathbf{3}}$ intervals would bring about a relatively large leap of $P_{2}$ toward $L_{2}$; lengthening the time would decrease the extent of jump. If no saltation occurred, both taps appeared in their "true" positions. The transition from "jump" to "no jump" does not occur abruptly, of course. Experience has dictated that the continuous variation in leaping distance should be observable over about a 60 -msec time change to warrant a positive "jump" report. All three observers carefully met this criterion (three switch stops).

$L_{1}$ remained throughout in the center of the palm; $L_{2}$ was moved systematically along one of eight radii 
separated from each other by 45-deg angles. Both ascending (saltation to no saltation) and descending series of the psychophysical method of limits, in 5$\mathrm{mm}$ steps, were employed. The criterion for a boundary was two successive reports in agreement past the point of change. Three practiced observers participated. For the most part, only a single radius could be completely explored in a single session (one ascending, one descending series). The demands of unvarying limb posture and the large number of time-interval sweeps needed to insure certainty, of judgment required this somewhat plodding approach. Nevertheless, two complete explorations of all eight radii were satisfactorily completed for each observer.

Results are displayed in Figure 2. The saltatory area of the palm proves to be more nearly circular in shape than any comparable area yet measured. Its average radius is $3.38 \mathrm{~cm}$, and its area approximates $31 \mathrm{~cm}^{2}$, about one-third of the entire palm. This is less than half that of the thorax or of the dorsal forearm. It is about $10 \%$ the size of the saltatory area of the ventral thigh. At the same time, it is considerably larger than anticipated from preliminary rough explorations of the hand and from certain theoretical considerations.

The latter are worthy of review. In the search for a principle of explanation that will account for the basic fact that there are well-defined barriers to the spread of the saltatory effect, that is, that definite, measurable saltatory areas exist, one must inevitably turn to the central nervous system. This, to be sure, is only a matter of belief, not proof, but the evidence, coming chiefly from visual saltation,

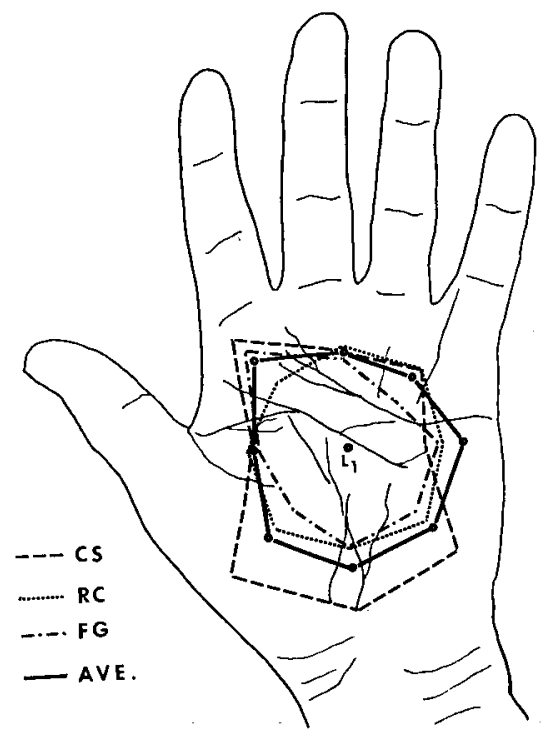

Figure 2. Saltatory area of the palm of the hand. The skin area within which saltation occurs covers about one-third of the total palm. strongly points to a central seat of the effect. Thus, the fact that a $P_{2}$ flash may be projected to one retina and $P_{3}$ presented to the corresponding hemisphere of the other, with continuity of saltation indiscriminable from single-hemiretina stimulation, demands a central explanation. Similarly, the fact that $L_{1}$ and $L_{2}$ may be positioned just outside the borders of the optic disk, with the result that clear hopping through the blind spot occurs (Lockhead, Johnson, \& Gold, 1980), permits no other interpretation. On the other hand, the saltatory effect is not achievable when $\mathrm{L}_{1}$ and $L_{2}$ are on opposite sides of the macula or when the cutaneous sites are separated by the body midline. Even $1-\mathrm{cm}$ separations at the midline of the forehead are not perceived as saltatory, despite the fact that such distances easily produce saltation when the sites are unilateral (Geldard, 1982, p. 159). This suggests that mechanical properties or neural organization of the periphery are not sufficient conditions for saltation.

\section{Pad of the Index Finger}

The palm having yielded a relatively small saltatory area, as contrasted with all other bodily regions previously explored, it was a natural extrapolation to extend sampling to a still smaller anatomical unit. The volar side of the left index finger was next selected for campimetric measurement. To reach it with two independently manipulable contactors, it was arranged to suspend the latter from counterweighted pendulums calibrated to deliver a force of $3 \mathrm{~g}$ at the fingertip. The finger pad could be brought to the contactors. This was accomplished by providing supports for the observer's elbow and forearm and a suitable Velcro strap that held the hand comfortably in place. Final precise adjustment of the two contactors was brought about by rack-and-pinion devices mounted on microscope supports. This arrangement permitted three-way regulation of contactor position. The contactors were mounted on $1.3-\mathrm{cm}$ Bimorph benders; each of these was supplied with a strain gauge that gave continuous monitoring on an oscilloscope. The same $1.8-\mathrm{mm}$-diam contactors as had been used on the palm were employed on the finger.

A spot in the middle of the finger pad was selected as Locus $1\left(\mathrm{~L}_{1}\right)$ to receive $\mathrm{P}_{1}$ and $\mathrm{P}_{2}$ while $\mathrm{P}_{3}$ explored, in 2.5-mm steps, eight radii $45 \mathrm{deg}$ apart, thus permitting excursion of $L_{2}$ over the entire volar tip of the finger. As in all previous experiments employing this general procedure, the method of limits in its conventional, unabridged form was used to determine the limits of saltatory leaping away from $L_{1}$. The observer manipulated the $P_{2}-P_{3}$ interval until the presence or absence of saltation was determined.

The same practiced observers participated in this experiment. Only two radii could be completed in a given experimental series without undue strain, so 
that several weeks of experimentation were needed to explore twice each of eight radii on the index fingers of three observers.

Individual and average results are displayed in Figure 3. It is, of course, immediately obvious that the pad of the index finger has a greatly restricted saltatory area. Indeed, the average area totals but $2.28 \mathrm{~cm}^{2}$ (it is $292.5 \mathrm{~cm}^{2}$ for the ventral thigh, $251.2 \mathrm{~cm}^{2}$ for the dorsal forearm, and $31.0 \mathrm{~cm}^{2}$ for the palm). Evidence from peripheral neural anatomy suggests that, in a general way, a rough negative correlation exists between density of innervation and size of saltatory area. This is an extremely important point, one to which we shall return.

\section{Volar Forearm}

Exploration of the dorsal forearm, reported earlier (Geldard, 1982, pp. 159-160), had been accomplished with somewhat larger contactors, and it seemed desirable, for purposes of comparison, to add the volar side of the forearm to the list of areas campimetrically covered. Accordingly, with the same three observers and with an apparatus replicating the essential conditions realized on the palm and finger, the left volar forearm of each of the observers was explored for the limits of saltation.

With a suitable padded armrest extending from a couch on which the observer lay supine, the volar side of the hand was approachable with contactors mounted on calibrated balance arms. The contactors were identical with those used on the finger and, like those, were mounted on 1.3-cm Bimorph benders. The balance arms supporting the latter were heavily weighted to prevent bounce and were calibrated to impart a force of $10 \mathrm{~g}$ at the skin. Each contactor assembly was adjustable in three dimensions by rackand-pinion and flexible arm arrangements.

In this experiment, $\mathrm{L}_{1}$ was centered laterally at a point midway between the crease of the elbow and that of the wrist. As with earlier explorations of the

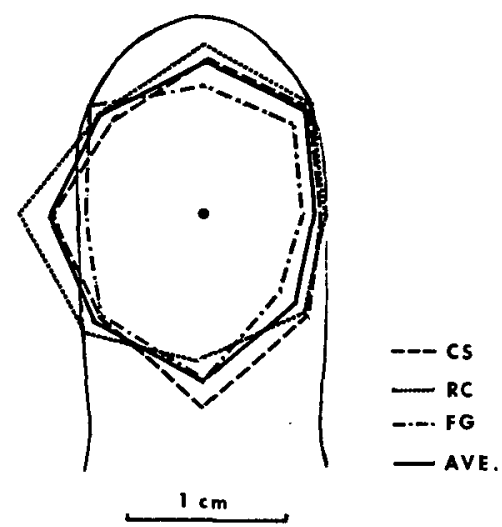

Figure 3. Saltutory area of the pad of the Index finger. Curvature of the surface is responsible, of course, for apparent extension of the saltatory boundaries past the projected outine of the digit.

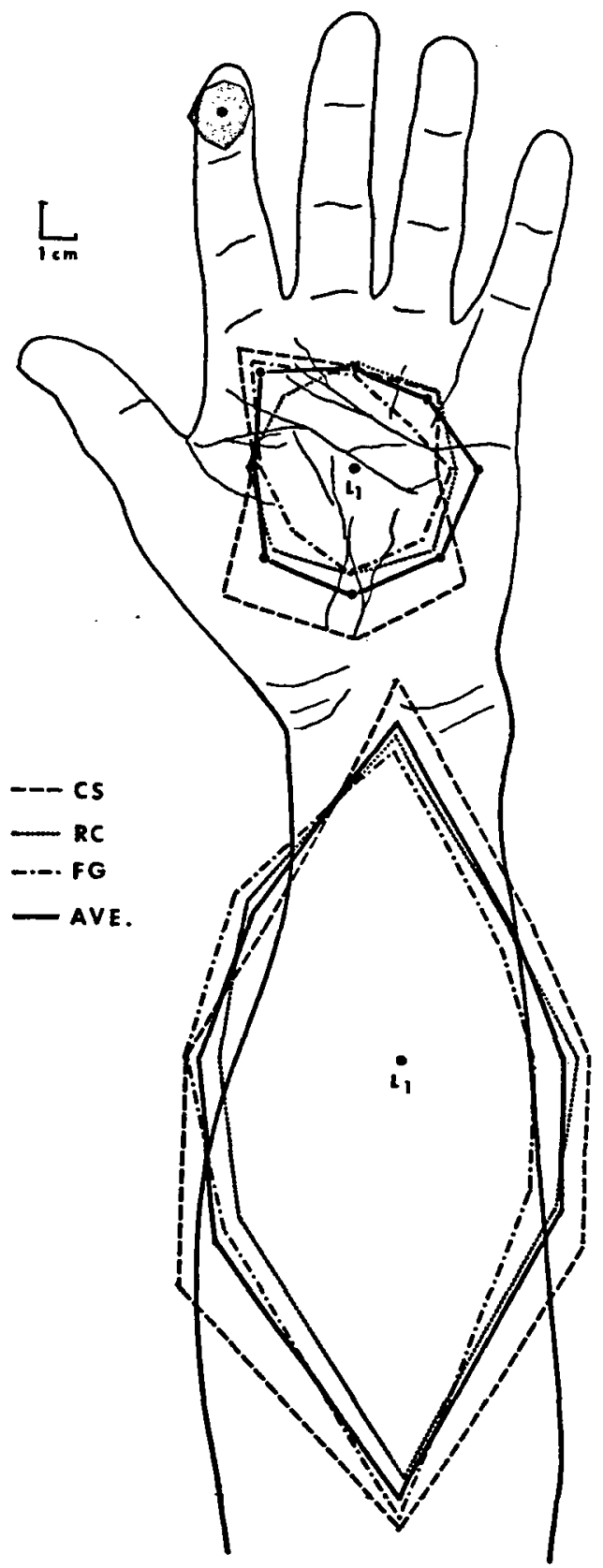

Figure 4. Saltatory area of the volar side of the forearm. Corresponding areas for palm and index finger are included for purposes of comparison.

palm and finger, eight radii were traversed by the conventional method of limits. Again, it was possible to complete only two ascending and descending series in a single observation period. However, three complete explorations on each of the three observers were completed in 36 experimental sessions.

The results are displayed in Figure 4, which for comparative purposes incorporates those obtained on the palm and finger as well as the forearm. The total saltatory area of the volar forearm proves to be $145.7 \mathrm{~cm}^{2}$ on the average, a value considerably 
smaller than that derived earlier for the dorsal forearm $\left(251.2 \mathrm{~cm}^{2}\right)$. Inspection of Figure 4 , since it makes apparent immediately the sizes and shapes of the three anatomical areas under consideration, reveals both similarities and differences. The chief similarity has to do with the shape of the saltatory area. Characteristically, it has been found that the long axis of the saltatory oval is parallel to the body or limb axis involved; transverse saltation is always less extensive. The major variation displayed in Figure 4 is, of course, the vast discrepancy in size of saltatory area over body loci, and this consideration leads to interesting speculation.

\section{Discussion}

We are learning much these days about central representation of peripheral events. For our purposes, we have the interesting maps of the cortex of the postcentral gyrus painstakingly arrived at through plotting of cortical receptive fields (Sur, Merzenich, \& Kaas, 1980). These show the quantitative relations obtaining between responsive fields of the monkey's skin and the cortical regions that represent them in Brodmann's areas $3 \mathrm{~b}$ and 1 of the contralateral parietal lobe. Cortical "magnification," a construct embraced by the formula cortical area/body area, is found to be low in the trunk, upper arm, and thigh regions but high in the hand and foot areas. Indeed, magnification is 100 times as great for the glabrous hand region of the owl monkey as for the trunk. Cortical receptive field size is linearly and inversely related to magnification. If we view the saltatory area as being determined by receptive field properties, we should expect comparisons of finger, palm, and forearm to yield pretty much what we have found. To a first approximation, the "magnification" rule is obeyed in the saltatory phenomenon.

Further correspondence of saltatory and receptive fields is evident in their common elliptical shape, noted above. This analog is further strengthened by the midline flattening found in exploring the ventral thorax (Geldard, 1982, Figure 14). An extraordinarily close correspondence is found with the thoracic receptive field of the cat (Rose $\&$ Mountcastle, 1959, Figure 9).

We can presume that the boundaries for the saltatory effect are not congruent with those of a single cortical receptive field, but rather with a statistical boundary representing some degree of overlap among multiple sets of such fields populating the region of the two loci. The exact degree of overlap required to produce a consistent judgment of saltation over the $P_{2}-P_{3}$ time intervals that we employed would be determined to some degree by the properties illustrated in the spatial maps and graphs derived by Sur et al. (1980, pp. 305ff).
The information provided by neurophysiological evidence tells us that the differences in saltatory areas among body sites correlates with receptive field sizes and shapes and that, within sites, the saltatory area must involve a large number of overlapping fields. The neurophysiological correlates of the phenomenon itself, however, are not forthcoming from any work of which we are aware. We are not dealing with a process that involves simple energy thresholds alone. The nearest obvious analog to our effect, at least in the time domain, is the phenomenon of backward inhibition or backward masking. The effects of backward masking on energy thresholds are short-lived, that is, extend perhaps $20 \mathrm{msec}$, whereas the present phenomenon extends to about $250 \mathrm{msec}$. When pattern detection is interfered with by an appropriate masker, however, the effects are temporally far-reaching, as Békésy (1971, p. 531) and Turvey (1973) found in vision and Craig (1982) in tactile pattern recognition. The saltation effect involves a pattern in the sense that each stimulus event can be characterized by intensitive, local, and pitch dimensions. These are to some degree separable, and the $P_{2}$ and $P_{3}$ pulses retain their identities until the time between them falls below 250 msec. At this point, the first detectable change is in the apparent location of $P_{2}$. As the time grows shorter, say to $100 \mathrm{msec}$, the location of $P_{2}$ shifts towards $L_{2}$, the intensity decreases somewhat, and the pattern becomes "duller." At coincidence, when the two taps merge spatially, the magnitude of the complex suddenly grows. The simple pattern perceived at the longer $\mathbf{P}_{2}-\mathbf{P}_{3}$ interval has thus undergone a continuous transformation.

What is common to both the saltatory and the masking paradigms is the phenomenon described by Békésy (1971, p. 571) when he reported an incorporation of one visual pattern by another as the time between them shifted. It is this construct that is often used to describe backward masking (see, e.g., Craig, 1982, p. 527), and in some ways appears to be the effect perceived in saltation. The second event does not simply inhibit the first, but rather incorporates it to produce a new pattern. In this respect, the phenomenon is akin to what Békésy (1958) described as funneling, that is, simultaneous inhibition and summation of patterns occupying interactive domains in space and time. (See also Ratliff, 1965.) Funneling is most strongly supported by empirical evidence in psychophysical studies and in research on peripheral neural mechanisms (see, e.g., Fiorentini \& Maffei, 1970), but Békésy (1967, p. 188) suggested that the construct could be applied to higher centers as well. In such cases, he held the opinion that the establishment of a funnel might be fairly rapid, but its disappearance could take up to a half-second. Considering the fact that several levels of neural 
activity may be required to represent the dimensions of the mechanical events of saltation, and that a number of cortical loci are known to be simultaneously active when a single peripheral event occurs, it may take some time and effort to uncover the exact conjugation of peripheral and central activities that saltation comprises.

\section{REFERENCES}

BÉ KÉsy, G. von. Funneling in the nervous system and its role in loudness and sensation intensity of the skin. Journal of the Acoustical Society of America, 1958, 30, 399-412.

Békésy, G. von. Sensory inhibition. Princeton, N.J: Princeton University Press, 1967.

BÉ KÉsy, G. voN. Auditory backward inhibition in concert halls. Science, 1971, 171, 529-536.

Craig, J. C. Vibrotactile masking: A comparison of energy and pattern maskers. Perception \& Psychophysics, 1982, 31, 523-529.

Fiorentini, A., \& Maffei, L. Transfer characteristics of excitation and inhibition in the human visual system. Journal of Neurophysiology, 1970, 33, 285-291.

GeldARD, F. A. Sensory saltation: Metastability in the perceptual world. Hillsdale, N.J: Erlbaum, 1975.
Geldard, F. A. Saltation in somesthesis. Psychological Bulletin, 1982, 92, 136-175.

Geldard, F. A., \& Sherrick, C. E. The cutaneous "rabbit": A perceptual illusion. Science, 1972, 178, 178-179.

Lockhead, G. R., Johnson, R. C., \& Gold, F. M. Saltation through the blind spot. Perception \& Psychophysics, 1980, 27, 545-549.

RATLIFF, F. Mach bands: Quantitative studies on neural networks. San Francisco: Holden-Day, 1965.

Rose, J. E., \& Mountcastle, V. B. Touch and kinesthesis. In J. Field, H. W. Magoun, \& V. E. Hall (Eds.), Handbook of physiology (Vol. 1) Neurophysiology. Washington, D.C: American Physiological Society, 1959.

Sherrick, C. E. The art of tactile communication. American Psychologist, 1975, 30, 355-360.

Sur, M., Merzenich, M. M., \& KaAs, J. H. Magnification, receptive-field area, and "hypercolumn" size in Areas 3b and 1 of somatosensory cortex in owl monkeys. Journal of Neurophysiology, 1980, 44, 295-311.

TuRvey, M. T. On peripheral and central processes in vision: Inferences from an information-processing analysis of masking with patterned stimuli. Psychological Review, 1973, 80, $1-52$.

(Manuscript received November 12, 1982; revision accepted for publication November 24, 1982.) 